\title{
Simulation of thermoacoustic waves by a pressure-based algorithm for compressible flows
}

\author{
Wei Li • Qi-Sheng Chen
}

Received: 1 June 2011 / Revised: 21 February 2012 / Accepted: 23 April 2012

(C)The Chinese Society of Theoretical and Applied Mechanics and Springer-Verlag Berlin Heidelberg 2012

\begin{abstract}
A modified SIMPLEC method which can solve compressible flows at low Mach number is introduced and used to study thermoacoustic waves induced by a rapid change of temperature at a solid wall and alternatingdirection flows generated by thermoacoustic effects in a tapered resonator. The results indicate that the algorithm adopted in this paper can be used for calculating compressible flows and thermoacoustic waves. It is found that the pressure and velocity in the resonator behave as standing waves, and the tapered resonator can suppress highfrequency harmonic waves as observed in a cylindrical resonator.
\end{abstract}

Keywords Compressible flow - Thermoacoustic wave . Resonator · SIMPLEC

\section{Introduction}

Algorithms for computational fluid dynamics (CFD) have been divided into two categories: the density-based method and the pressure-based method. The former, which treats the set of governing equations in a coupled way, is usually used for compressible flows and the latter refers to a segregated algorithm, developed for incompressible flows. The ratio of the flow speed to the speed of sound in the fluid (the Mach number) determines whether exchange between kinetic energy of the motion and internal degrees of freedom needs to be considered [1]. Actually, compressible and incompressible flows are mathematically different, and are distinguished by their respective hyperbolic and elliptic governing equations systems. Some

The project was supported by the National Natural Science Foundation of China (50890182, 10972226).

W. Li · Q.-S. Chen $(\bowtie)$

Key laboratory of Microgravity,

Institute of Mechanics, Chinese Academy of Sciences,

15 Beisihuan West Road, 100190 Beijing, China

e-mail: qschen@imech.ac.cn problems such as poor convergence and deteriorated accuracy occur when dealing with incompressible flows by adopting technologies developed for compressible flows directly. However, the compressible effects need to be considered in many practical problems even with a very low fluid speed, such as flows involving a strong heat source and flows generated by some thermoacoustic effects.

With preconditioning, the density-based methods can be used to solve low-Mach-number flows [2-5]. But this technology confronts an obvious problem of computational stability, especially for viscous flows. This problem has been discussed by Turkel [6] and Lee [7] and is considered difficult to overcome. Meanwhile many researchers devote themselves to extending methods designed for incompressible flows to the compressible regime [1, 8-10].

In this paper, we present a modified SIMPLEC method which can solve compressible flows at low Mach number and use this method to study the thermoacoustic waves induced by a rapid change of temperature at a solid wall and alternating-direction flows generated by thermoacoustic effects in a resonator.

\section{Numerical strategy}

The continuity, momentum, energy and state equations for compressible ideal gas are written as

$\frac{\partial \rho}{\partial t}+\nabla \cdot(\rho \boldsymbol{V})=0$

$\frac{\partial(\rho \boldsymbol{V})}{\partial t}+\nabla \cdot(\rho \boldsymbol{V} \boldsymbol{V})=-\nabla p+\nabla \cdot(\mu \nabla \boldsymbol{V})+\frac{1}{3} \nabla(\mu \nabla \boldsymbol{V})$,

$\frac{\partial(\rho e)}{\partial t}+\nabla \cdot(\rho e \boldsymbol{V})=\boldsymbol{S}:(\nabla \boldsymbol{V})-p \nabla \cdot \boldsymbol{V}+\nabla \cdot(k \nabla T)$,

$p=\rho R T$,

where $\mu$ and $k$ are the dynamic viscosity and the thermal conductivity, respectively. The internal energy per unit mass, $e=C_{V} T, C_{V}$ is the specific heat at constant volume, and $R$ is the specific gas constant. $S$ is the viscous part of the stress tensor. 
In this paper, the SIMPLEC algorithm [1] is extended by correcting density and velocities simultaneously in the pressure-correction equation with the help of state equation. The mass flux correction at the e face of a control volume $(\mathrm{CV}), P$, can be expressed as

$\dot{m}_{\mathrm{e}}^{\prime}=\left(\rho^{*} S v_{\mathrm{n}}^{\prime}\right)_{\mathrm{e}}+\left(v_{\mathrm{n}}^{*} S \rho^{\prime}\right)_{\mathrm{e}}$,

where $\rho^{\prime}$ and $v_{\mathrm{n}}^{\prime}$ represent corrections of the density and the contravariant velocity (i.e., the velocity normal to the CV face), $S$ is the area of the e face. The first term in Eq. (5) refers to the gradient of pressure correction, $p^{\prime}$, and can be considered as a diffusive term in the pressure-correction equation

$v_{\mathrm{n}, \mathrm{e}}^{\prime}=-\Delta \Omega\left(\frac{1}{A_{\mathrm{P}}^{v_{\mathrm{n}}}}\right)_{\mathrm{e}}\left(\frac{\delta p^{\prime}}{\delta n}\right)_{\mathrm{e}}$,

where $A_{\mathrm{P}}$ is the coefficient of $v_{\mathrm{n}}$ in the algebraic equations for velocities, and $\Delta \Omega$ is the volume of the control volume. The second term in Eq. (5) is due to the compressibility and involves the correction to density at the $\mathrm{CV}$ face. It can be expressed in terms of the pressure correction as

$\rho^{\prime}=\left(\frac{\partial \rho}{\partial p}\right)_{\mathrm{T}} p^{\prime}=\frac{p^{\prime}}{R T}$.

This term needs to be handled as a convective term.

The governing equations are discretized using the control volume method. We use the deferred correction approach for the estimation of flow variables at the e face of a control volume

$F_{\mathrm{e}}=F_{\mathrm{e}}^{\mathrm{L}}+\left(F_{\mathrm{e}}^{\mathrm{H}}-F_{\mathrm{e}}^{\mathrm{L}}\right)^{\mathrm{old}}$,

where $F_{\mathrm{e}}^{\mathrm{L}}$ stands for the approximation of convective and diffusive fluxes by a lower-order scheme, and $F_{\mathrm{e}}^{\mathrm{H}}$ is the higherorder approximation. The term indicated by the superscript "old" is evaluated explicitly. The convective flux at the CV face is evaluated using the SMART scheme [11], and the diffusion flux is estimated by a second-order central scheme. For the time integration, we choose a fully implicit three time level scheme

$\left(\frac{\partial \phi}{\partial t}\right)^{n+1} \approx \frac{1.5 \phi^{n+1}-2 \phi^{n}+0.5 \phi^{n-1}}{\Delta t}$,

where the superscript denotes the time level.

\section{Results and discussions}

\subsection{Thermoacoustic waves in a nitrogen-filled cavity}

Thermoacoustic waves can be generated in a nitrogen-filled cavity by rapidly heating or cooling a sidewall. When a part of compressible fluid is rapidly heated (cooled), this part of fluid expands (contracts) and gives rise to a fast increase (decrease) in the local pressure. The local pressure perturbations lead to the occurrence of pressure waves, which are called thermoacoustic waves. The propagation of thermoacoustic waves is simulated in the cavity as shown in Fig. 1.

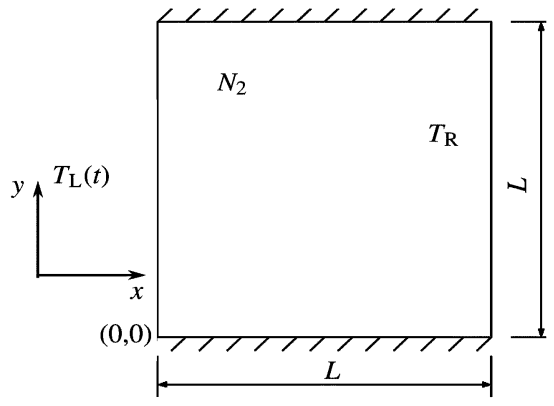

Fig. 1 Geometry and boundary conditions of a cavity where thermoacoustic waves are generated

The fluid is maintained at a constant temperature $T_{0}=$ $300 \mathrm{~K}$ and pressure $p_{0}=0.1 \mathrm{MPa}$. The horizontal walls are thermally insulated. On the left sidewall of the cavity is imposed a time-dependent temperature, and the right side wall is kept at a constant temperature

$$
\begin{aligned}
& T_{\mathrm{L}}(t)=T_{0}+A T_{0}\left(1-\mathrm{e}^{-t / \tau_{\mathrm{h}}}\right), \\
& T_{\mathrm{R}}=T_{0},
\end{aligned}
$$

where $A$ is the overheat ratio, $\tau_{\mathrm{h}}=C \cdot \tau_{\mathrm{c}}$, is a time constant indicating the speed of temperature change at the left sidewall, and $C$ is the time-constant coefficient. $\tau_{\mathrm{c}}=L / c_{0}$, is the travel time of sound waves over the length of the cavity, and $c_{0}$ denotes the sound speed at the initial condition.

To avoid non-physical oscillations at boundaries [12], the densities at the walls are treated with a transport equation derived from the continuity equation [13],

$\frac{\partial \rho}{\partial t}+\frac{1}{c} \frac{\partial p}{\partial n}+\rho \frac{\partial V}{\partial n}=0$,

where the local sound speed, $c=\sqrt{(\partial p / \partial \rho)_{\mathrm{s}}}, n$ is the normal pointing to the fluid, and the subscript " $\mathrm{s}$ " denotes the isentropic process.

For the mesh independence verification, we study a case under three different grids with $401 \times 51,501 \times 61$ and $601 \times 71$ and a time step $\Delta t=3.6 \times 10^{-8} \mathrm{~s}$. The overheat ratio is $A=0.33$ and $C=0.1$. When we use a grid with $501 \times 61$ control volumes, the curve of the pressure versus time at the center of cavity does not change under further refined grid (Fig. 2).

The thermoacoustic waves are generated inside the cavity by the rapid temperature change at the left sidewall. Figure 3 depicts the pressure and velocity variations with time at the middle point of the cavity with different overheat ratios, $A=0.1,0.33$ and 1.0 , and $C=0.1$. The first pressure peak corresponds to the thermoacoustic wave which propagates forward inside the cavity and the second pressure peak corresponds to the thermoacoustic wave which is reflected from the right sidewall of the cavity. The amplitudes of the pressure oscillation and velocity oscillation at the middle point increase with the overheat ratio (Fig. 3). The thermoacoustic 
waves have a very sharp front and a long tail of decay, which is similar to those observed by Brown and Churchill [14] in their experiments.

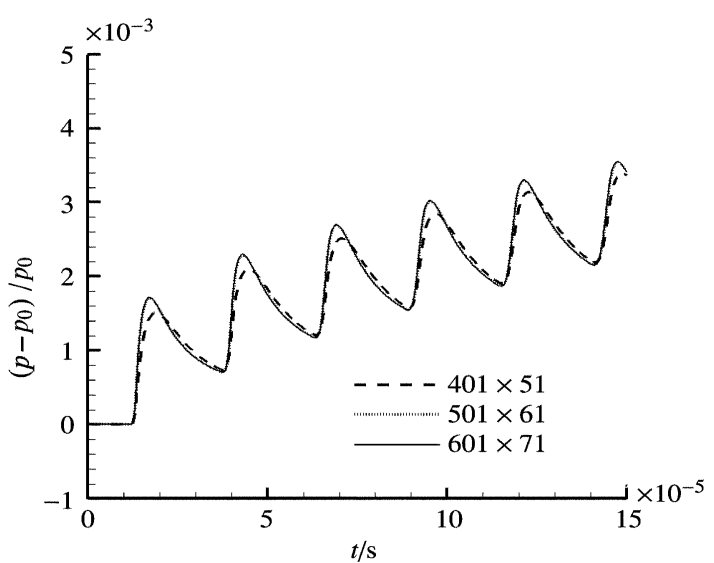

Fig. 2 Pressure evolvements with time at the center of cavity with different grids. The curves with grids of $501 \times 61$ and $601 \times 71$ overlap
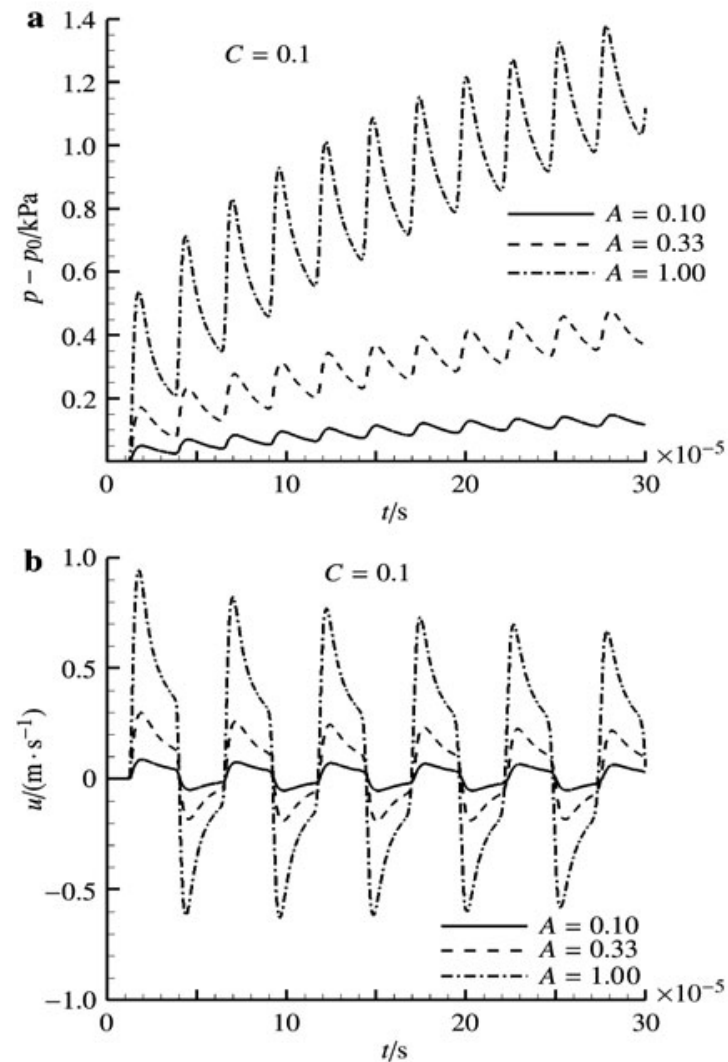

Fig. 3 Variations of a pressure and $\mathbf{b}$ velocity with time at the middle point of the cavity with different overheat ratios

When the left sidewall is rapidly cooled thermoacoustic waves can also be produced. Figure 4 shows the variations of pressure and velocity with time at the middle point of the cavity with a negative overheat ratio $A=-0.33$ and $C=0.1$.
There is a phase shift of pressure and velocity at the middle point of the cavity as compared to the variations shown in Fig. 3. Figure 5 shows variations of pressure with time at the middle point of the cavity with $A=0.33$ and different time-constant coefficients, $C=0,0.1,0.5,1$ and 3 . When the time-constant coefficient increases, the amplitude of the thermoacoustic wave decreases. A bigger time constant leads to
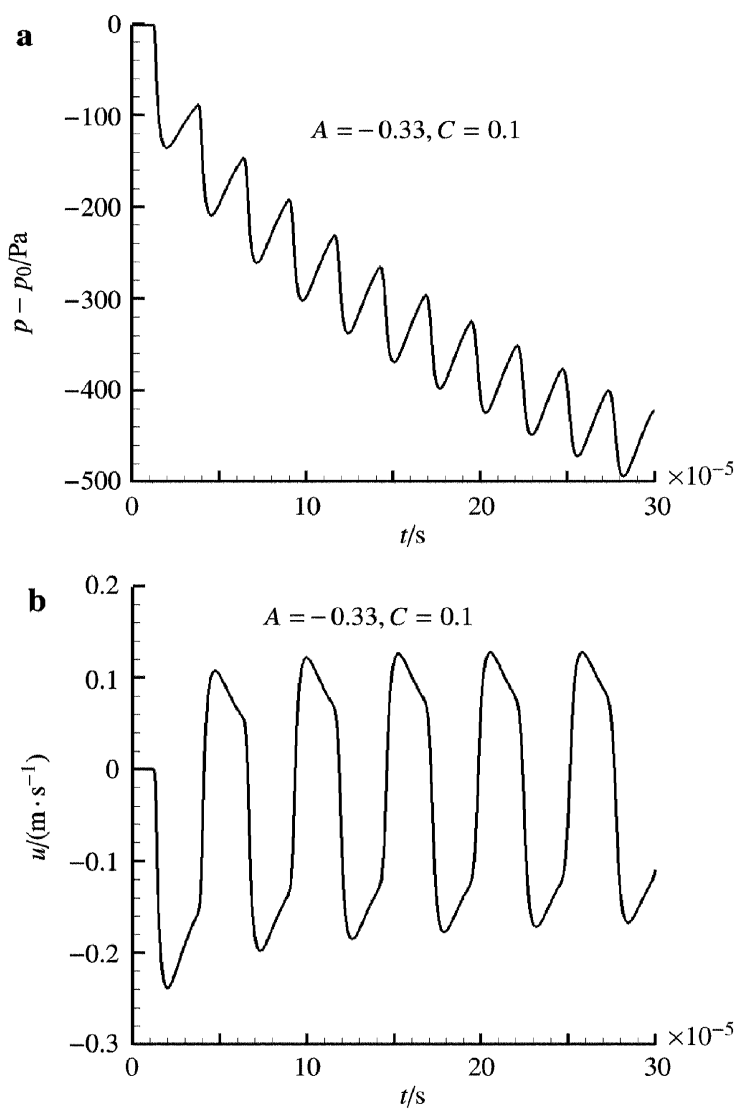

Fig. 4 Variations of a pressure and $\mathbf{b}$ velocity with time at the middle point of the cavity with a negative overheat ratio

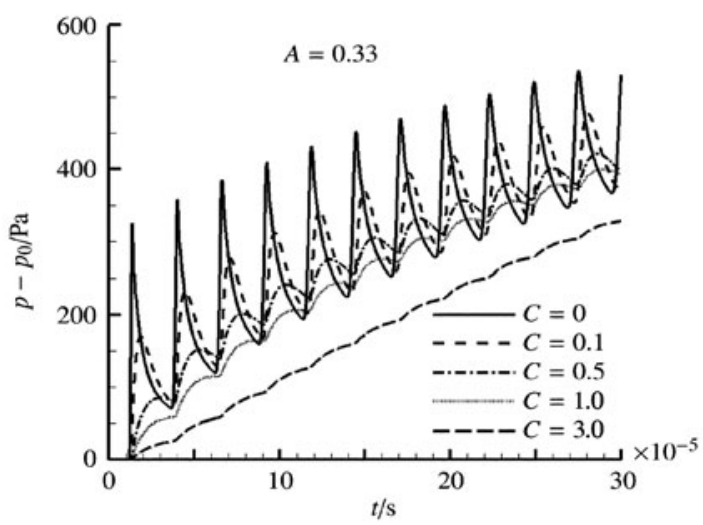

Fig. 5 Variations of pressure with time at the middle point of the cavity with different time-constant coefficients 
a slower temperature change at the left sidewall, and the energy input from left sidewall would diffuse into the surroundings rather than generate thermoacoustic waves.

3.2 Alternating-direction flows in a large-size tapered resonator

Thermoacoustic waves can be generated in a thermoacoustic engine. Here we consider compressible flows in a large-size tapered resonator as shown in Fig. 6. The initial pressure is, $p_{0}=30 \mathrm{bar}$, and temperature is $300 \mathrm{~K}$. A time-dependent pressure at the inlet of the resonator is imposed,

$p(t)=p_{0}+A_{\mathrm{p}} \sin (2 \pi f t)$,

where $A_{\mathrm{p}}$ and $f$ are the amplitude of pressure oscillation and the frequency at the inlet of the resonator respectively.

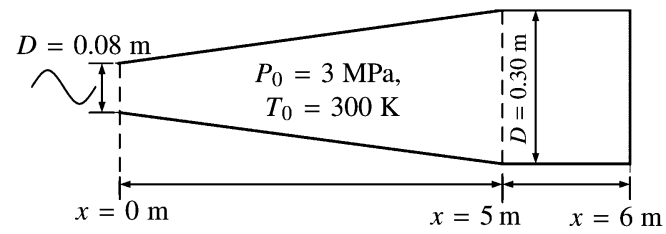

Fig. 6 Configuration of a large-size resonator

A grid with $482 \times 32$ is used for calculating waves and the time step is $\Delta t=1.5 \times 10^{-5} \mathrm{~s}$. A pressure sensor was arranged at the position $x=5.1 \mathrm{~m}$ in the experiment [15]. Local pressure ratio $p_{\max } / p_{\min }$ can be obtained by the Fourier analysis. Table 1 shows the comparison of pressure ratios between computational and experimental results at different average pressures and pressure ratios at the inlet. The computational results agree well with the experimental results.

Table 1 Comparison between computational and experimental results [15]

\begin{tabular}{lccc}
\hline & $\begin{array}{c}\text { Ambient } \\
\text { Pressure/MPa }\end{array}$ & $\begin{array}{c}\text { Pressure ratio } \\
\text { at the inlet }\end{array}$ & $\begin{array}{c}\text { Pressure ratio } \\
\text { at } x=5.1 \mathrm{~m}\end{array}$ \\
\hline Ref. [15] & 2.971 & 1.0629 & 1.0115 \\
Current result & 3.0 & 1.0629 & 1.0109 \\
Ref. [15] & 2.983 & 1.0975 & 1.0177 \\
Current result & 3.0 & 1.0975 & 1.0185 \\
Ref. [15] & 3.01 & 1.1241 & 1.0225 \\
Current result & 3.0 & 1.1241 & 1.0237 \\
Ref. [15] & 3.045 & 1.1698 & 1.0304 \\
Current result & 3.0 & 1.1698 & 1.0311 \\
\hline
\end{tabular}

The pressure and velocity histories are probed at points $x=0,3,4$ and $5 \mathrm{~m}$ and $r=0.02 \mathrm{~m}$ in the calculations. Fig- ure 7 shows that there is a nearly $180^{\circ}$ phase-shift of pressure between the locations $x=3$ and $x=4 \mathrm{~m}$, but the phase shift does not appear for axial velocities at the same points (Fig. 8). The pressure leads the velocity by a phase angle of $90^{\circ}$ at the point $x=3 \mathrm{~m}$ while the pressure leads the velocity by a phase angle of $-90^{\circ}$ at $x=4 \mathrm{~m}$ (Fig. 9).

We define the volumetric flow rate as, $U=\int_{\mathrm{S}} \boldsymbol{V} \cdot \boldsymbol{n} \mathrm{d} S=$ $A_{\mathrm{U}} \sin (2 \pi f t+\phi)$, where $A_{U}$ and $\phi$ are the amplitude and phase of $U$, respectively. Amplitudes of the pressure oscillation and volumetric-flow-rate oscillation along the axis are drawn in Fig. 10 for different pressure ratios at the inlet, $p_{\max } / p_{\min }=1.0408,1.0629,1.0975,1.1241,1.1487$ and 1.1698 . The pressure node locates at about the position $x=3.4 \mathrm{~m}$ where the amplitude of pressure oscillation is almost zero and the amplitude of volumetric-flow-rate oscillation reaches a maximum at approximately the same position.

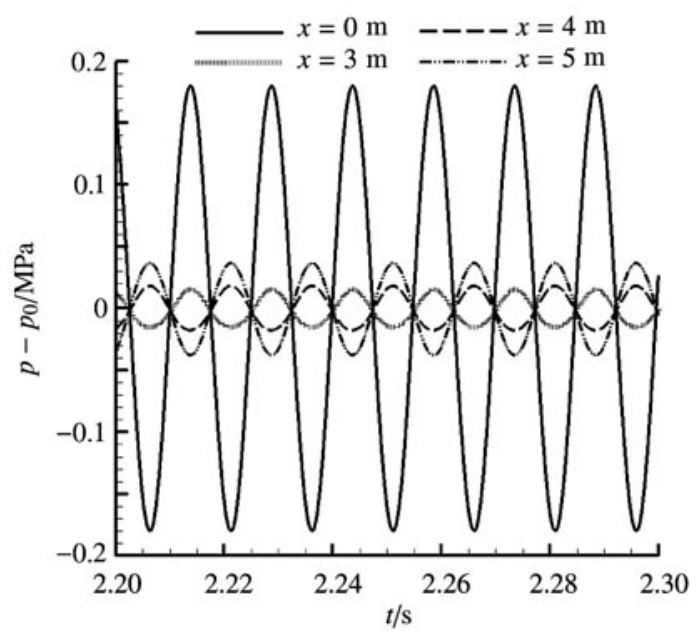

Fig. 7 Curves of pressure histories at different points

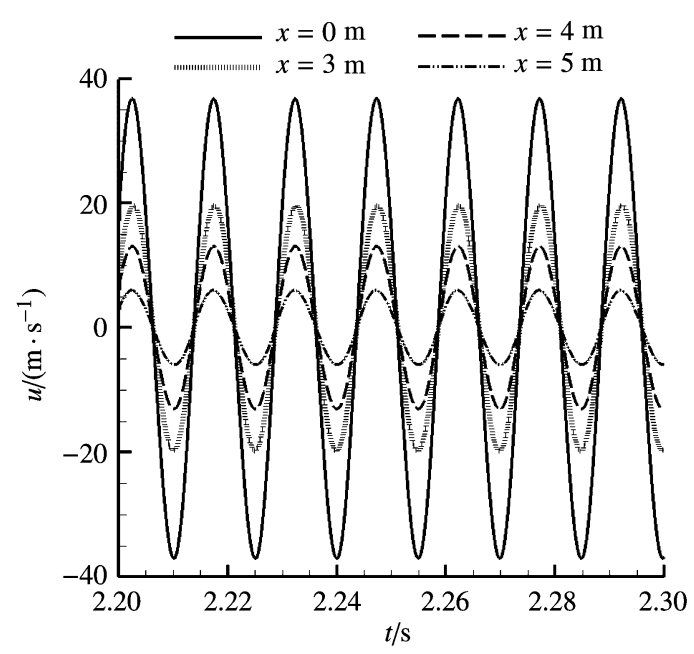

Fig. 8 Curves of axial velocity histories at different points 

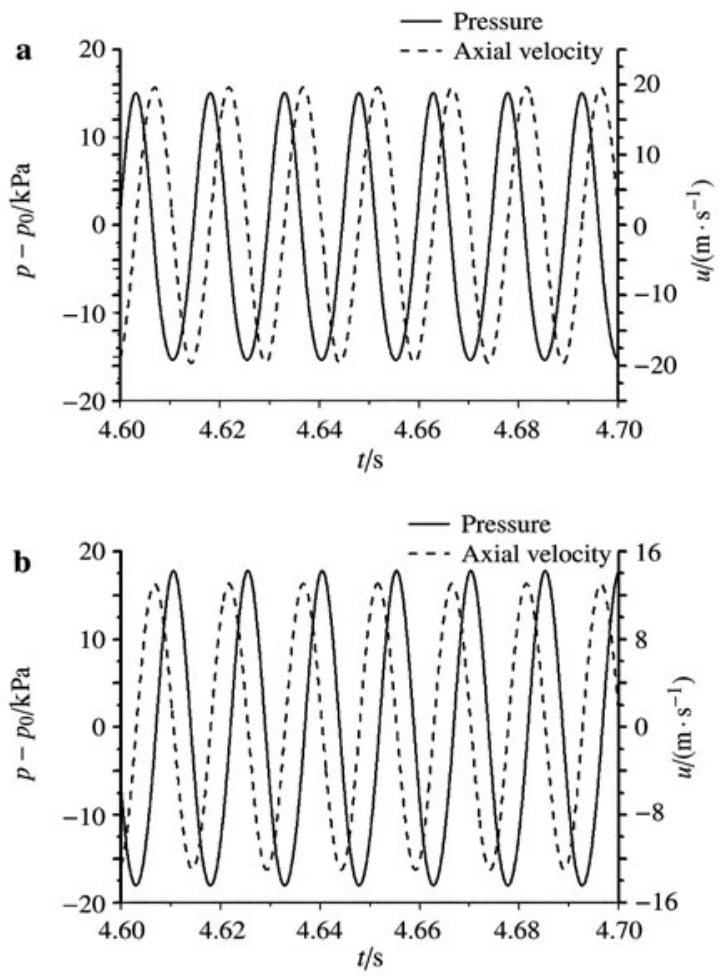

Fig. 9 The phase shifts between the pressure and axial velocity at points $\mathbf{a} x=3 \mathrm{~m}$ and $\mathbf{b} x=4 \mathrm{~m}$
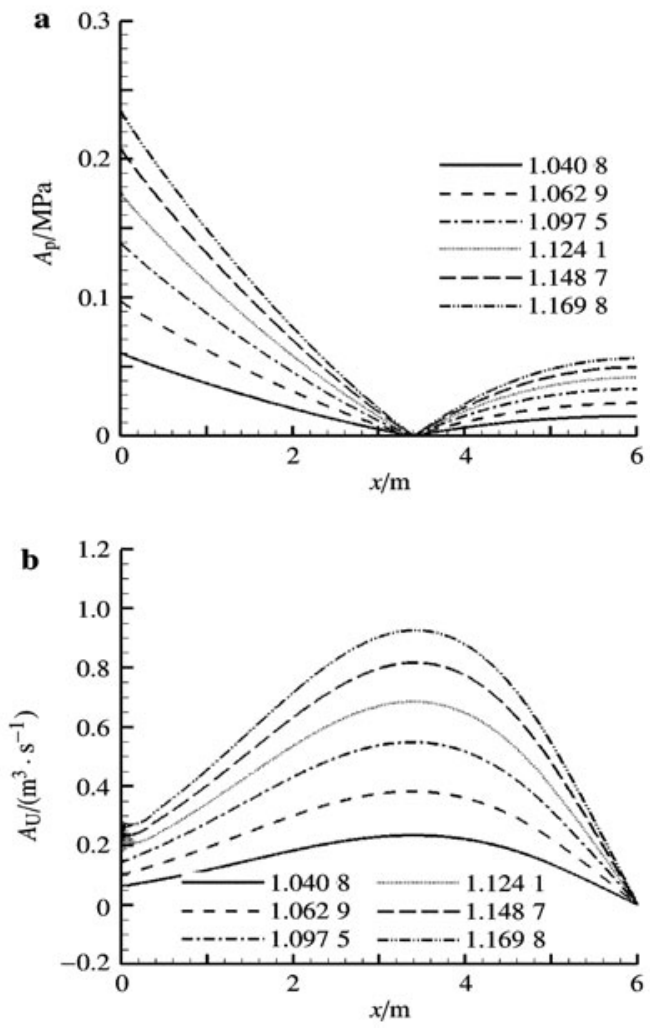

Fig. 10 a Amplitude of pressure oscillation and $\mathbf{b}$ amplitude of volumetric-flow-rate oscillation along the axis for different pressure ratios at the inlet
The appearance of the pressure node is caused by superposition of the incoming pressure wave and the reflected wave from the end of the resonator.

Fourier analyses of volumetric flow rate at different points show that there is only one frequency in the spectrum at $x=3 \mathrm{~m}$ and $x=5 \mathrm{~m}$ (Fig. 11). Zaripov et al. [16] observed the occurrence of shock wave caused by harmonics at high frequencies in a cylindrical pipe in which oscillation was excited by a reciprocating piston. The suppression of high-frequency harmonics in the tapered-shape resonator can be explained by two factors. The first one has been discussed in Ref. [17] through the analysis of the nonlinear term in the following one-dimensional inviscid momentum equation for compressible gas,

$$
\frac{\partial u}{\partial t}+u \frac{\partial u}{\partial x}=-\frac{1}{\rho} \frac{\partial p}{\partial x} .
$$

Using a tapered resonant cavity can significantly decrease the axial velocity and velocity gradient so that the nonlinear term $u \partial u / \partial x$ can be decreased. The other is related to the fact that a specific resonator shape exhibits a unique resonance modal spectrum which can affect the amplitude and phase of harmonics of a standing wave excited in the resonator [18]. The tapered-shape resonator is also called energy-focused resonator.
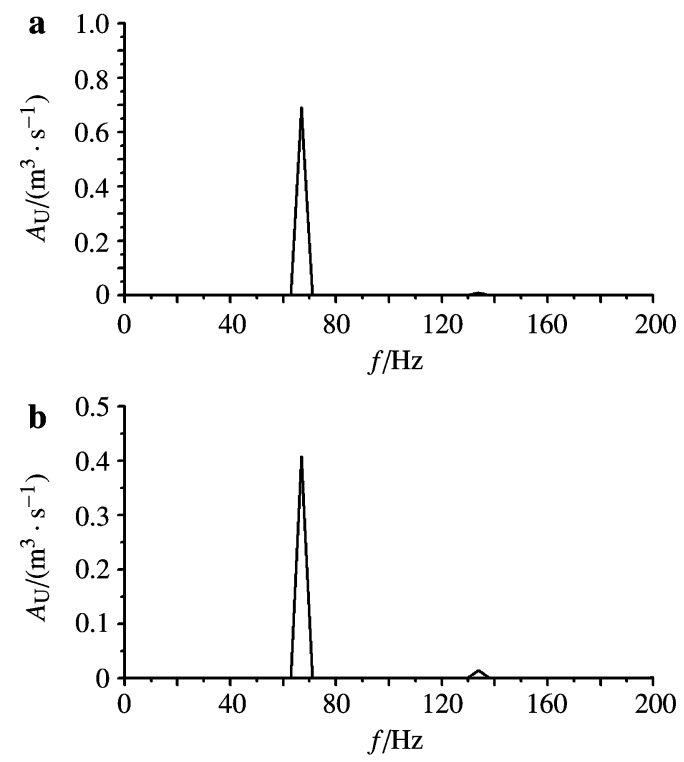

Fig. 11 Fourier analyses of volumetric flow rate at points with a $x=3 \mathrm{~m}, \mathbf{b} x=5 \mathrm{~m}$

\section{Conclusions}

We present here an algorithm which solves the pressurecorrection equation for compressible flows to satisfy the continuity equation. The algorithm is used to calculate thermoacoustic waves in a nitrogen-filled cavity. It is found that 
the amplitude of pressure oscillation inside the cavity increases with the overheat ratio. When the time-constant coefficient increases, the amplitude of the thermoacoustic wave decreases.

Alternating-direction flows inside a large-size tapered resonator are also simulated. The computed pressure oscillation amplitudes and pressure ratios compare well with the experimental results. In the resonator, the pressure and the velocity behave as standing waves, and there is a pressure node at the position $x=3.4 \mathrm{~m}$ for different pressure ratios at the inlet. The results indicate that the tapered resonator can suppress higher-frequency harmonics as observed in the cylindrical pipe. The algorithm presented here can be used for solving compressible-flows involving hydrodynamic waves.

\section{References}

1 Ferziger, J. H., Peric, M.: Computational Methods for Fluid Dynamics, Springer, German (2002)

2 Turkel, E.: Preconditioned methods for solving the incompressible and low speed compressible equations. Journal of Computational Physics 72, 277-298 (1987)

3 Weiss, J. M., Smith, W. A.: Preconditioning applied to variable and constant density flows. AIAA Journal 33, 2050-2057 (1995)

4 Edwards, J.R., Liou, M.S.: Low-diffusion flux-splitting methods for flows at all speeds. AIAA Journal 36, 1610-1617 (1998)

5 Liou, M. S.: A sequel to AUSM, Part II: AUSM+-up for all speeds. J. Comput. Phy. 214, 137-170 (2006)

6 Turkel, E.: Preconditioning techniques in computational fluid dynamics. Annual Reviews of Fluid Mechanics 31, 385-416 (1999)
7 Lee, S. H.: Cancellation problem of preconditioning method at low mach numbers. J. Comput. Phy. 225, 1199-1210 (2007)

8 Shyy, W., Chen, M. H.: Pressure-based multigrid algorithm for flow at all speeds. AIAA J. 30, 2660-2669 (1992)

9 Moukalled, F., Darwish, M.: A high-resolution pressure-based algorithm for fluid flow at all speeds. Journal of Computational Physics 168, 101-133 (2001)

10 Nerinckx, K., Vierendeels, J., Dick, E.: Mach-uniformity through the coupled pressure and temperature correction algorithm. Journal of Computational Physics 206, 597-623 (2005)

11 Darwish, M. S., Moukalled, F.: Normalized variable and space formulation methodology for high-resolution schemes. Numerical Heat Transfer B 26, 79-96 (1994)

12 Farouk, B., Oran, E. S., Kailasanath, K.: Numerical simulation of the structure of supersonic shear layers. Physics Of Fluids A-Fluid Dynamics 3, 2786-2798 (1991)

13 Poinsot, T. J., Lele, S. K.: Boundary conditions for direct simulations of compressible viscous flows. Journal of Computational Physics 101, 104-129 (1992)

14 Brown, M. A., Churchill, S. W.: Experimental measurements of pressure waves generated by impulsive heating of a surface. AIChE Journal 41, 205-213 (1995)

15 Yu, G. Y., Luo, E. C., Dai, W.: An energy-focused thermoacoustic-Stirling heat engine reaching a high pressure ratio above 1.40. Cryogenics 47, 132-134 (2007)

16 Zaripov, R. G., Ilhamov, M. A.: Non-linear gas oscillations in pipe. Journal of Sound and Vibration 46, 245-257 (1976)

17 Luo, E. C., Ling, H., Dai, W..: A high pressure ratio energyfocused thermoacoustic heat engine with a tapered resonator. Chinese Science Bulletin 50, 284-286 (2005)

18 Lawrenson, C. C., Lipkens, B., Lucas, T. S., et al.: Measurement of macrosonic standing waves in oscillating closed cavities. J. Acoust. Soc. Am. 104, 623-636 (1998) 